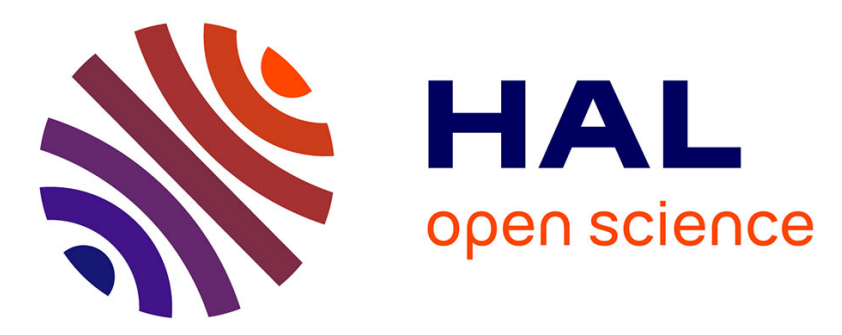

\title{
Recherche en finance: quand la performativité invite à la réflexivité
}

\author{
Hélène Rainelli, Hélène Rainelli-Weiss
}

\section{To cite this version:}

Hélène Rainelli, Hélène Rainelli-Weiss. Recherche en finance: quand la performativité invite à la réflexivité. Gérer et Comprendre. Annales des Mines, 2019, 135 (1), pp.29-41. 10.3917/geco1.135.0029. halshs-02025011

\section{HAL Id: halshs-02025011 https://shs.hal.science/halshs-02025011}

Submitted on 19 Feb 2019

HAL is a multi-disciplinary open access archive for the deposit and dissemination of scientific research documents, whether they are published or not. The documents may come from teaching and research institutions in France or abroad, or from public or private research centers.
L'archive ouverte pluridisciplinaire HAL, est destinée au dépôt et à la diffusion de documents scientifiques de niveau recherche, publiés ou non, émanant des établissements d'enseignement et de recherche français ou étrangers, des laboratoires publics ou privés. 


\title{
Recherche en finance : quand la performativité invite à la réflexivité
}

\author{
Par Hélène RAINELLI-WEISS \\ IGR-IAE, Université de Rennes 1, CREM - UMR 6211
}

\begin{abstract}
Cet article se propose de faire le bilan de l'impact de la recherche en finance sur ces soixante dernières années. II tente de mesurer l'influence des théories sur les pratiques et la croissance de l'industrie financière mais aussi sur les normes sociales et les arrangements institutionnels qui organisent le monde de la finance. Notre principale contribution est d'insister sur le rôle de la théorie dans la construction sociale du monde financier et sur la nécessité pour la théorie financière de cesser d'ignorer ses effets performatifs, afin de s'inscrire, de manière plus ambitieuse, dans les débats sur l'utilité sociale de la finance et de tracer des perspectives novatrices, notamment en matière de régulation financière.
\end{abstract}

\section{Introduction}

La finance, ses acteurs et ses institutions se trouvent, depuis le début de la crise financière des années 2007-2008, au centre de débats nourris sur le bien-fondé de pratiques que l'exposition médiatique a amenées au grand jour. Entre amendes records payées par les grandes banques internationales aux différents régulateurs et procès retentissants de traders poursuivis en justice par leur employeur ou par les autorités publiques, c'est la légitimité de toute une industrie, longtemps florissante et en pleine expansion, qui se trouve mise en cause. Les enseignants et chercheurs en finance ne sont pas épargnés par cette vague de fond, et ce d'autant moins qu'ils exercent parfois, aux États-Unis notamment, les fonctions de conseillers en matière de politiques économiques, voire de gouverneurs de banques centrales. On se souvient des explications embarrassées de certains professeurs américains dans le documentaire Inside Job consacré aux responsabilités dans la crise financière. Fait notable car inédit, en 2015, le professeur Luigi Zingales ouvre le congrès annuel de l'American Finance Association 1, qu'il préside, sur la question de savoir si la finance contribue au bien-être social (Zingales, 2015) : constatant le divorce grandissant entre l'industrie financière et l'opinion publique, il s'interroge sur le rôle des producteurs de savoir et sur les améliorations que la situation d'alors appelle.

Ce contexte particulier, où la crise financière semble générer une crise morale (Munir, 2011 ; Orlean, 2009), fournit un excellent prisme pour réfléchir à la question de l'impact de la recherche en finance sur les pratiques de l'industrie financière et sur la société plus généralement. Sur une période de cinquante ou soixante ans, la

\footnotetext{
${ }^{1}$ L'American Finance Association (AFA) est l'association académique phare de la recherche financière mondiale. Elle se donne pour objectif le développement et la promotion du savoir en économie financière.
} 
recherche en finance, partie de presque rien, a conquis des sommets de visibilité, valu des prix Nobel d'économie à une demi-douzaine de ses représentants, établi une forme de domination comme champ intellectuel dans le domaine des sciences de gestion (Whitley, 1986), avant de se voir accusée d'être au moins en partie responsable de la crise financière de ces dernières années ou des évolutions négatives du capitalisme contemporain (Pryke et Allen, 2000 ; Li Puma et Lee, 2004 ; Aglietta et Rebérioux, 2004 ; Artus et al., 2008 ; Bryan et Rafferty, 2006 ; Bryan et Rafferty, 2014 ; Davis, 1994 ; Zingales, 2015). Cette situation singulière permet d'adopter une perspective rétrospective potentiellement riche d'enseignements et aussi d'interrogations sur la manière d'appréhender, de mesurer ou de juger « l'impact » sur la société en général d'un certain type d'effort intellectuel visant à construire une science (ici, de la finance). Elle invite à distinguer plusieurs formes d'impact et à tenter de comprendre comment ces formes s'articulent entre elles.

C'est ce que le présent article se propose de faire dans les développements qui suivent. Nous décrirons tout d'abord l'impact direct que la théorie financière a eu, dès son origine, sur les pratiques de l'industrie financière d'une part et sur la théorie ellemême, c'est-à-dire dans le processus cumulatif visant à construire un paradigme, une vision du monde apte à fournir un cadre cognitif commun aux acteurs du monde de la finance. Nous montrerons ensuite, en mobilisant la notion de performativité, que l'impact direct sur les pratiques se traduit en réalité par une influence forte, et souvent imprévue, des théories financières sur la réalité sociale à la transformation de laquelle elles participent de fait. Pour illustrer la puissance de ces processus de transformation de la réalité sociale, nous prendrons des exemples issus de la régulation financière, qui constitue le terrain privilégié pour articuler théorie financière et recherche du bien social. Enfin, nous montrerons que ces phénomènes de construction sociale de la réalité par la théorie, repérés dans d'autres disciplines ou par les acteurs du débat social, ne sont absolument pas pris en compte par la recherche en finance à ce jour. Nous analyserons les manques que crée cette situation et la manière dont d'autres disciplines s'emparent du défi ainsi créé. L'essentiel de notre contribution sera de proposer une réflexion sur les connaissances produites par ces autres disciplines et sur les bénéfices qu'il y aurait pour la théorie financière à entrer en dialogue avec elles. Cela lui permettrait de prendre une part moins limitée dans les débats de société que suscitent les pratiques de l'industrie financière et de se réapproprier l'ambition de contribuer de manière à la fois plus novatrice et plus agissante à la construction du bien-être social.

\section{L'impact direct de la recherche en finance}

Que la recherche en finance ait exercé depuis les travaux de Markowitz (1952) une très forte influence sur les pratiques des financiers, notamment de marché, est un phénomène notable (MacKenzie, 2006). Peter Bernstein (1995), qui fut gestionnaire de fonds puis président d'une entreprise de consultants pour l'industrie financière, est probablement le premier à en avoir rendu compte systématiquement. Son portrait d'une poignée d'universitaires en aventuriers de la finance moderne, initiateurs d'une révolution théorique sans précédent, n'oublie pas de préciser que ces derniers, " conquis par la Bourse », ont, pour la plupart, " été associés à un moment ou à un autre, à une firme de Wall Street ou à une grande société d'investissement » (Bernstein, 1995 : 18 ; voir aussi MacKenzie et Spears, 2014 ; Charreaux et Albouy, 
2017). L'hommage qu'il leur rend est sans ambiguïté : « Les contributions de ces innovateurs (les fondateurs de la finance moderne, parmi lesquels Bachelier, Markowitz, Sharpe, Leland, etc.) ont eu un impact profond sur notre vision des choses, malgré les controverses qu'elles ont soulevées au moment de leur apparition » (Bernstein, 1995 : 298). De fait, aucun gestionnaire de fonds aujourd'hui ne travaille hors du cadre rentabilité/risque établi par Markowitz (1952) puis Sharpe (1964). Le principe de diversification, qu'on leur doit, est devenu la base de tous les raisonnements financiers. De la même façon, le recours aux produits dérivés pour gérer le risque selon les préceptes du modèle de Black et Scholes (1973) est devenu une norme universelle (Martin, 2015 ; MacKenzie et Millo, 2003).

Répondant aux besoins pratiques de la gestion de portefeuilles ou de l'évaluation des actifs financiers, en prise avec les activités de marchés à Wall Street ou dans l'industrie financière (Bernstein, 1995), les fondateurs de la finance moderne ont ainsi non seulement accompagné mais aussi favorisé les transformations de l'industrie financière (Chiapello et Walter, 2016). Ils ont largement contribué à développer de nouvelles activités dont les principales sont sans doute de nouvelles formes de gestion de fonds (gestion indicielle et stratégies d'assurances de portefeuilles, création de fonds spécialisés dans l'arbitrage) ou de management des risques. Concernant ce dernier domaine, il est ainsi patent que le développement de gigantesques marchés " hors marchés », les fameux marchés OTC de dérivés (marchés over-the-counter ou de gré à gré dont l'encours en montant notionnel atteint 542000 milliards de dollars en juin 2017, contre 33000 milliards pour les dérivés échangés sur les marchés boursiers ${ }^{2}$ ), repose entièrement sur les possibilités presque infinies d'innovations financières offertes par le modèle de Black et Scholes (1973) (Huault et Rainelli, 2009 ; MacKenzie, 2006). Sans la technologie proposée par ce modèle, véritable matrice théorique pour des développements ultérieurs, jamais de tels encours n'auraient pu être atteints et jamais l'activité d'intermédiation des banques dans ces activités n'aurait pu se développer au rythme où elle l'a fait (Huault et Rainelli, 2009 ; Lépinay, 2011 ; Morgan, 2008 ; MacKenzie et Spears, 2014).

Si c'est en praticien de la finance que Bernstein rend hommage à la recherche académique des années 1950-1990, Arnold et al. (2003) éclairent, pour leur part, le bilan académique des recherches en finance. Leur méthode consiste à analyser l'impact des articles les plus connus sur les recherches ultérieures, ce qui est une manière de mesurer le caractère cumulatif de la connaissance produite dans la discipline.

Arnold et al. (2003) classent ainsi les cinquante articles en finance les plus cités dans les principales (et plus prestigieuses) revues académiques de la discipline (Journal of Business, Journal of Finance, Journal of Financial Economics, Journal of Finance and Quantitative Analysis, Review of Financial Studies, Financial Management). Ils montrent que les articles les plus souvent cités en finance sont à la source de processus cumulatifs actifs au cours desquels ils sont mobilisés par de nombreux chercheurs dont l'objectif est d'étendre ou d'améliorer les résultats initialement obtenus. Parmi ces " articles-sources », se distinguent, dans l'étude d'Arnold et al. (2003), les papiers de Jensen et Meckling (1976) et de Black et Scholes (1973), dont

\footnotetext{
2 Source : BIS Statistical Bulletin, juin 2017.
} 
il est noté qu'au-delà du cercle de la finance académique dont ils sont issus, leur influence s'étend très largement en direction d'autres sciences sociales. Une utilisation sommaire de Google Scholar renforce cette dernière conclusion. Les auteurs des principales théories de la finance moderne font décidément "référence ». Markowitz (1952), Sharpe (1964), Black et Scholes (1973) et Jensen et Meckling (1976) sont cités respectivement $23000,17000,30000$ et 56000 fois dans des revues académiques qui dépassent largement le seul cadre de la recherche en finance.

On notera que les auteurs évoqués jusqu'ici, les pères fondateurs de la finance moderne, sont tous issus des États-Unis, et que l'influence de leur pensée sur les développements de la finance porte la trace à la fois de la puissance économique des États-Unis pendant la période considérée (1950-1990) et des spécificités institutionnelles de ce pays. Le financement de l'économie par les marchés financiers de tradition aux États-Unis, mais aussi les changements historiques liés à l'évolution des conditions économiques, des institutions légales et des conceptions sur ce que doit être une entreprise et comment elle doit être contrôlée (Fligstein, 1993) ont abouti, dans ce pays, à l'émergence d'une conception de l'entreprise fondée sur la valeur actionnariale (Fligstein, 1993 ; Zorn, 2004 ; Zorn et al., 2014) qui s'appuyait sur les développements théoriques de la théorie de l'agence (Jensen et Meckling, 1976) et de l'efficience des marchés. Le rôle prééminent du pays dans l'économie mondiale explique, pour partie, la diffusion de ces conceptions dans l'ensemble des pays développés.

Mais la perspective rétrospective sur les développements de la finance académique depuis soixante ans ne permet pas seulement de prendre la mesure du caractère cumulatif de la connaissance produite par les chercheurs de la théorie financière moderne. Elle incite aussi à appréhender cette discipline comme proposant un paradigme unique, très cohérent, et dont les bases n'ont jamais été remises en cause jusqu'à très récemment (Rainelli-Le Montagner, 2003 ; Zingales, 2015). Myers (2015) illustre cette cohérence interne de la finance lorsqu'il revient sur sa contribution à la constitution de ce paradigme, tout en revendiquant l'impact du manuel co-écrit avec Brealey - « le » célèbre Brealey and Myers, best-seller mondial des manuels de finance depuis sa première édition en 1981, dont il affirme qu'il a " substantiellement amélioré la pratique financière »-, et en soulignant son rôle en tant qu'expert de l'évaluation ou de la détermination des taux d'actualisation auprès des autorités publiques. Au fond, la théorie financière moderne structure les débats sur la finance depuis environ soixante ans. Elle sert de référence aux décideurs tant publics que privés et constitue un tout cohérent, à l'épistémologie plus complexe qu'il n'y paraît (Rainelli-Le Montagner, 2003 ; De Scheemaekere, 2009), mais qu'il convient de qualifier de " paradigme " au sens de Kuhn (1972). On peut noter que des années 1950 au milieu des années 1990 au moins, la théorie financière n'a cessé de développer ce paradigme original, traitant des anomalies rencontrées selon la procédure typique des phases de science " normale » (Kuhn, 1972), où les fondements du paradigme (théorie des anticipations rationnelles et efficience des marchés dans le cas d'espèce) ne sont pas discutés. L'attribution du prix Nobel d'économie à Fama en 2013 vient couronner cet achèvement, même si la récompense devait être partagée avec Shiller, grand critique de la théorie de l'efficience des marchés. 
Au total, l'impact direct de la théorie financière moderne apparaît très important à la fois sur les pratiques et sur l'appréhension intellectuelle des phénomènes financiers. Si la recherche en finance semble influente et même triomphante jusqu'à la dernière crise financière, c'est qu'elle peut se targuer d'avoir enclenché avec succès un processus intellectuel cumulatif, résultant d'une longue construction temporelle en phase (et aussi parfois en tension) avec les attentes des praticiens de marchés et les évolutions technologiques de l'industrie (dématérialisation des échanges et big data notamment). Jouant un rôle indispensable dans le fonctionnement du système financier global à tous les niveaux y compris ceux de la régulation et de la comptabilité financière (Merton, 1995 :14), la théorie financière semble constituer un cas particulier parmi les autres sous-disciplines du management. En marketing par exemple, bien que les outils développés par la recherche académiques soient utilisés par les praticiens, le taux de pénétration de la recherche dans les pratiques paraît largement inférieur à ce qui se constate en finance (Roberts et al., 2014). En recherche organisationnelle, le questionnement est encore plus fondamental. Davis (2015) compare ce champ académique à une curiosité touristique située à San José en Californie : la maison Winchester des mystères, un manoir de type victorien de cent soixante pièces, pleine de détails architecturaux sans usage pratique. Des portes qui ouvrent sur des murs, des couloirs labyrinthiques qui ne mènent nulle part, des escaliers montant jusqu'au plafond : telle est l'image qu'il utilise pour peindre la discipline de la recherche organisationnelle où, souligne-t-il, la recherche de résultats constamment nouveaux et intéressants semble détourner les auteurs académiques de la recherche de la vérité, au détriment du caractère cumulatif de la connaissance produite et de son impact sur les pratiques. En comptabilité/contrôle enfin, la division entre recherche académique et implications pratiques semble plus marquée qu'en finance. Cette division a fait l'objet de débats récents (Kaplan, 2011 ; Parker et al., 2011 ; Baldvinsdottir et al., 2010) d'où il ressort que, pour certains, la recherche en comptabilité s'éloigne trop fortement des préoccupations des professionnels. Une étude menée aux États-Unis a ainsi révélé que si cette recherche a bien une influence sur les pratiques, c'est essentiellement à travers le rôle qu'elle joue dans la formation des étudiants, et très peu à travers un impact direct qu'elle aurait sur les professionnels (Moehrle et al., 2009).

La finance académique peut ainsi être considérée comme entretenant avec la pratique une relation particulière, sans équivalent dans les autres sous-disciplines du management. Ceci n'est pas sans conséquences sur l'influence qu'elle exerce, souvent de manière imprévue et par des processus à la fois complexes et émergents, sur les transformations de la finance telle qu'elle est pratiquée et régulée dans les sociétés contemporaines.

\section{La performativité de la théorie financière}

Le fait que les théories financières aient eu et continuent d'avoir une influence massive sur les pratiques relève à la fois de l'évidence et de l'impensé chez les chercheurs de la finance académique. Tenue pour acquise, cette influence renforce leur sentiment personnel d'utilité sociale, mais ne les conduit presque jamais (Zingales, 2015 ; Shiller, 2012, font ici, figure d'exception) à interroger la contribution au bien-être social des pratiques financières qu'ils ont concouru à établir et à légitimer. Plus fondamentalement, ni la manière dont cette influence s'exerce ni les conséquences imprévues qu'elle peut avoir ne font l'objet d'une attention particulière 
dans ce champ scientifique. Ces questions surgissent parfois ailleurs, notamment dans le débat public qui s'organise autour des crises financières majeures. Elles trouvent alors la théorie financière à peu près muette et tout à fait désarmée. On se souvient d'Alan Greenspan concédant en 2008 que le krach lui a ouvert les yeux sur les faiblesses du raisonnement économique et l'a laissé dans un "état d'incrédulité et de choc ", devant l'effondrement d'un pilier central de la théorie des marchés libres. On se souvient aussi qu'il affirmait « ne pas comprendre exactement pourquoi c'[était] arrivé ${ }^{4}$.

Hors du champ de la finance académique, ou à ses marges, quelques auteurs ont cependant pointé (Rainelli-Le Montagner, 2003 ; MacKenzie, 2006 ; Bryan \& Rafferty, 2006) et commencé à étudier (MacKenzie et Millo, 2003 ; MacKenzie, 2006 ; Martin, 2015 ; MacKenzie et Spears, 2014 ; Huault et Rainelli, 2009 ; RainelliWeiss et Huault, 2016) les processus par lesquels la théorie financière co-construit les phénomènes qu'elle s'attache à décrire. Dans l'effort de dévoiler ces processus, la notion protéiforme de performativité (MacKenzie et Millo, 2003 ; Callon 1998 ; Cabantous et Gond, 2011 ; Gond et al., 2015), utilisée dans des acceptions variables par les chercheurs en sciences de gestion (Gond et al., 2015 ; Abrahamson et al., 2016) aussi bien que par des philosophes, des linguistes, des sociologues des sciences (par exemple Cabantous et Gond, 2011 ; Harding, 2003 ; Learmonth, 2005 mais aussi Derrida, 1979 ; Searle, 1969 ; Butler, 1997 ou Callon, 2007), se révèle particulièrement utile. Pour illustrer l'intérêt de ce concept, retenons ici, parmi d'autres possibles, la perspective qui s'astreint à observer et à tenter de comprendre comment les théories façonnent la « réalité », jusqu'à, parfois, la faire advenir (Barnes, 1983 ; Pickering, 1995 ; Callon, 1998 ; Mackenzie et Millo, 2003). Dans cette perspective, il s'agit d'analyser les impacts souvent émergents, et non envisagés par la théorie elle-même, de l'application des cadres cognitifs qu'elle promeut, sur le monde des pratiques réelles. Ferraro, Pfeffer et Sutton (2005) ont identifié et décrit trois mécanismes qui rendent compte des processus à l'œuvre dans ce type de performativité. Nous proposons de les reprendre ici pour illustrer la manière dont la théorie financière participe à la construction des arrangements institutionnels qui préside à l'organisation des marchés financiers, instaure des normes sociales et façonne un vocabulaire qui influence durablement la façon dont les individus se représentent la réalité financière des marchés et des entreprises. C'est à dessein que les exemples pris ici concernent spécifiquement la régulation financière, qui constitue un espace privilégié pour observer la rencontre entre théorie financière et recherche du bien-être social.

\section{Les arrangements institutionnels}

La théorie qui a sans doute eu l'impact le plus important, le plus global et le plus visible sur les institutions de la finance moderne est la théorie de l'efficience des marchés financiers. Décrite il y a quarante ans par Jensen (1978) comme établie ("There is no other proposition in economics which has more solid empirical evidence supporting it."), cette théorie est beaucoup plus discutée depuis que deux brèches

\footnotetext{
${ }^{4}$ Audition du 23 octobre 2008 au congrès américain devant le House Committee on Oversight and Government Reform.
} 
importantes lui ont été portées. La première est celle introduite par les tenants de la finance comportementale qui ont apporté de nombreuses preuves empiriques portant sur les biais comportementaux des investisseurs sur les marchés financiers (Broihanne, Merli et Roger, 2004 ; Orléan, 2009). La seconde brèche a été portée par la répétition et l'ampleur des crises financières qui se sont succédé depuis la moitié des années 1990, avec une régularité qui correspond bien moins aux prédictions de la théorie de l'efficience des marchés qu'à l'expérience historique sur période longue de ces phénomènes (Kindelberger, 2001).

Quoi qu'il en soit de sa validité réelle et de son acceptation dans la communauté des chercheurs aujourd'hui (Zingales, 2015), il n'en demeure pas moins que la théorie de l'efficience des marchés a eu un effet très important sur le développement et sur la régulation des marchés et notamment sur ceux des marchés OTC. Jusqu'à la crise des subprimes, les chercheurs en finance se sont réjouis de la puissance de leurs outils théoriques qui permettaient aux grandes banques d'investissement notamment, en utilisant la matrice proposée par le modèle de Black et Scholes, de proposer des innovations financières nombreuses et variées (Morgan, 2008 ; Huault et Rainelli-Le Montagner, 2009 ; Lépinay, 2011 ; Martin, 2015). Jusqu'aux subprimes, il ne venait à l'esprit de personne d'envisager que ces innovations pouvaient aussi avoir une face sombre et être utilisées par exemple pour duper les investisseurs moins informés (Zingales, 2015). La bienveillance dont bénéficiaient ces innovations de la part de l'ensemble de la communauté financière mais aussi, globalement, des régulateurs, a permis que se développent d'énormes marchés fort peu régulés, les marchés OTC évoqués plus haut. Toute réflexion sur la régulation possible de ces marchés partait du postulat qu'il fallait, en tout état de cause, éviter de freiner, par la régulation, l'activité sur ces marchés et au contraire promouvoir, sous toutes ses formes, cette même activité (Huault et Rainelli-Weiss, 2013). Ce qui sous-tend ce postulat, c'est l'idée, directement issue de la théorie de l'efficience des marchés, que l'échange libre est ce qui produit des marchés efficaces et remplissant correctement leur fonction. Plus acheteurs et vendeurs peuvent se livrer à l'échange sans entrave, plus l'information privée qu'ils détiennent se reflète dans les prix des actifs financiers et devient accessible aux autres investisseurs. Dans le paradigme de la théorie de l'efficience des marchés, il n'y a d'activités de marché que souhaitables, puisque ce sont elles qui génèrent des marchés à même de jouer pleinement leur rôle de dispensateurs de signaux fiables aux acteurs économiques (Orléan, 2009). Les débats sur la régulation (Rainelli-Weiss et Huault, 2016), c'est-à-dire sur l'organisation des marchés OTC, se centrent ainsi naturellement sur les moyens de garantir cette efficience par l'octroi de la plus grande liberté aux acteurs de ces marchés. Il est évidemment paradoxal de noter ici que, développés presque sans entrave au nom même de la théorie de l'efficience des marchés, qui rejoignait par ailleurs les théories d'évaluation des actifs financiers prônant la " complétude " des marchés et accueillant de ce fait toute innovation avec enthousiasme, les marchés OTC se caractérisent jusqu'à aujourd'hui par leur manque de transparence, l'arrangement institutionnel construit autour d'eux ne faisant pas obligation aux vendeurs qui y agissent d'afficher des prix publics à destination des acheteurs ${ }^{5}$. On a ainsi laissé croître des marchés censés dispenser des signaux fiables aux acteurs privés et publics, sans trop se soucier de l'opacité dans laquelle ces signaux étaient

\footnotetext{
${ }^{5}$ Cette situation est en train d'évoluer, notamment en Europe, avec l'entrée en vigueur, le 3 janvier 2018, de la directive MiFID 2.
} 
émis et sans analyser les sources importantes de profit que cette opacité offrait aux grandes banques d'investissements qui dominent ces marchés (Rainelli-Weiss et Huault, 2016). Notons que ce cas permettrait de revenir sur les débats qui intéressent les spécialistes de la performativité (MacKenzie, 2003, 2006 ; Cabantous et Gond, 2011 ; Gond et al., 2015 ; Muniesa, 2014), puisqu'on voit à travers lui l'écart qui sépare théorie performative, c'est-à-dire agissant sur le réel, et théorie autoréalisatrice. Ce n'est pas parce que la théorie de l'efficience influence fortement le type de régulation choisi jusqu'à très récemment pour organiser les marchés OTC que ces marchés deviennent pour autant « efficients ». Mais cela n'invalide en rien le constat selon lequel la théorie contribue à façonner ces marchés. On fait bien face à un ensemble théorique qui « provoque », selon le terme de Muniesa (2014), une réalité qui, sans elle, aurait pu prendre une forme bien différente.

\section{Les normes sociales}

Une plateforme privilégiée pour observer l'influence indirecte de la théorie financière sur les normes sociales est fournie par les obligations juridiques imposées aux gestionnaires de fonds. Aux États-Unis, comme au Canada et plus généralement dans le droit anglo-saxon, ces obligations sont dites fiduciaires (fiduciary duty) et se trouvent au confluent de deux logiques institutionnelles : celle du droit, d'une part, qui se doit de protéger les avoirs confiés aux gestionnaires de fonds et celles de la théorie financière, puisque ces gestionnaires y puisent les prescriptions nécessaires concernant leur tâche d'investisseurs sur les marchés financiers (Lydenberg, 2014). Ces deux logiques institutionnelles ne reposent pas forcément sur des conceptions identiques du comportement approprié. Lydenberg (2014) souligne ainsi l'intéressant hiatus entre les concepts de comportement " raisonnable », usuellement attendu en droit et de comportement « rationnel », hérité de l'économie néoclassique par la théorie financière moderne. Le comportement raisonnable en matière de fiducie a longtemps été défini comme un comportement prudent, sage, reposant sur le jugement et l'intelligence personnels, soucieux des conséquences des investissements décidés sur le monde réel. Sous l'influence de la théorie financière moderne, notamment de sa part relative à la gestion de portefeuille (Lydenberg, 2014), cette norme de comportement « raisonnable » a peu à peu été remplacée par une norme de comportement rationnel (au sens défini précisément par Markowitz dans les années 1950), visant la maximisation de la richesse des individus ayant confiés leurs fonds à des gestionnaires, en charge de leur fournir les plus grands profits possibles.

La différence est d'importance si on note, à la suite de Lydenberg (2014), que la définition d'un comportement " raisonnable " comme norme souhaitable repose sur une référence à la manière dont les individus agissent usuellement en société et se conforment à des standards et à des principes acceptés par la majorité. Le prudent man des obligations fiduciaires traditionnelles est donc celui qui agit « avec le soin, l'expertise, la prudence et la diligence que mettrait un homme prudent agissant avec une capacité comparable et familier de ce type d'affaires, à entreprendre une action de ce type et avec un but comparable » (US Code 29 ch. 18, § 1104 (a) (1) (B)). La référence au comportement « raisonnable » implique ainsi une certaine conception de la norme sociale et de la raison, et est associée à des traditions de philosophie morale qui interrogent les concepts d'équité, de justice et de bien commun. L'individu 
rationnel de la tradition économique et, par extension, de la tradition financière, lui, agit dans son intérêt propre. Aussi large et non égoïste soit ce dernier, il ne dépend pas d'une norme sociale, ni de principes largement partagés. II s'agit d'un intérêt personnel, individuel, défini sans référence aux autres, qui relève principalement de la philosophie utilitariste et mobilise les notions d'efficience, de maximisation de l'utilité, et de bien-être privé.

Ainsi le remplacement normatif, en termes d'obligations fiduciaires, du comportement raisonnable par le comportement rationnel pourrait, selon certains auteurs (Lydenberg, 2014), expliquer l'inconfort grandissant des investisseurs qui doutent que les critères de rentabilité et de risque suffisent toujours à définir leur satisfaction (qu'on songe à la naissance et au développement de l'investissement socialement responsable, ou au conflit entre objectif financier et maintien de l'emploi pour un investisseur salarié face à une OPA hostile). II aurait aussi fait passer par pertes et profits d'autres obligations fiduciaires plus larges, comme celles qui se soucieraient de l'impact des investissements financiers sur l'économie réelle ou de la répartition juste des profits entre les générations.

Quoi qu'il en soit, pendant toute la première moitié du $X X^{e}$ siècle aux États-Unis, c'est bien la référence à un comportement raisonnable qui a présidé à la définition des obligations fiduciaires des gestionnaires de fonds. Tout change dans la seconde moitié du $X X^{e}$ siècle sous l'effet des transformations de l'industrie financière rendues possibles par les innovations théoriques (gestion de portefeuille, trading de produits dérivés, confiance dans l'efficience des marchés) (Lydenberg, 2014).

L'investissement prudent, défini comme la sélection avisée de supports variés d'investissement basée sur le jugement des gestionnaires de fonds, est remplacé par l'investissement contrôlant correctement les risques selon les principes de la théorie financière. Dans les années 1990, les textes de lois évoluent. Le gestionnaire de fonds n'est plus requis d'agir en homme prudent mais en investisseur prudent, c'està-dire en investisseur appliquant les principes de la théorie moderne de portefeuille. Ce faisant, le standard de l'investisseur rationnel plutôt que raisonnable s'impose, justifié par la théorie financière et permettant, par son caractère normatif, d'éviter les comportements de corruption ou les conflits d'intérêt dont la possibilité avait grandi avec les montants des fonds gérés, notamment sous l'effet du développement sans précédent des fonds mutuels et des fonds de retraite. II ne s'agissait plus tant d'agir dans l'intérêt des bénéficiaires des fonds que dans l'intérêt de la performance économique desdits fonds en termes de rentabilité et de risque, changement qui doit tout à la théorie financière (Lydenberg, 2014).

L'analyse proposée par Montagne (2012) de l'évolution de la définition du principe de prudence, qui est au cœur des obligations fiduciaires aux États-Unis, permet de prendre, à un niveau d'analyse encore plus fin, la mesure de l'influence de la théorie financière sur les normes sociales du fiduciary duty. Elle montre en particulier comment le principe de prudence est d'abord timidement influencé par la théorie moderne de portefeuille dans le droit américain, à une époque où celle-ci n'est pas encore complètement acceptée par l'ensemble des experts. Elle met en évidence la manière dont le temps joue pour la théorie moderne de portefeuille à laquelle les juges et les avocats seront amenés à faire plus fréquemment référence au fur et à mesure que cette dernière s'instaure comme un standard dans les formations à la finance. Peu à peu (Montagne, 2012 :106), les juges se mettent à appuyer 
l'ensemble du raisonnement juridique les ayant conduits à une décision donnée sur la théorie de l'efficience des marchés, ce qui, sans les amener à prendre des décisions univoques, donne à cette dernière une influence très centrale sur la définition de normes sociales et légales d'importance pour les acteurs concernés.

À travers cet exemple des obligations fiduciaires, on voit l'influence de la théorie financière sur la redéfinition de normes sociales et juridiques en matière de comportement souhaitable pour un gestionnaire de fonds. Cette influence dépasse la simple redéfinition de pratiques mais s'inscrit profondément dans le paysage normatif et juridique. Même si les juges conservent des marges d'interprétation (Montagne, 2012), il est clair que la théorie impacte leurs décisions et modifie dès lors en profondeur les comportements dans l'industrie financière.

\section{Le vocabulaire}

La notion de fair value (juste valeur), qui s'est imposée - parmi les standards comptables internationaux (IFRS) adoptés dans les pays développés dans la décennie 2000 - comme méthode de valorisation des actifs financiers, se définit comme la valeur à laquelle un actif pourrait être vendu lors d'une transaction normale entre intervenants sur les marchés. Comme méthode de comptabilisation, elle s'oppose à la valorisation au coût historique qui a longtemps prévalu dans les normes comptables nationales, notamment françaises, et n'a pu s'imposer qu'au prix d'un processus contradictoire largement documenté dans la littérature en comptabilité (Power et Miller, 2013). Poussée par un petit groupe de régulateurs comptables affranchis des pratiques et des institutions professionnelles habituelles (Power et Miller, 2013), la fair value fut d'emblée critiquée pour son manque de réalisme issu des hypothèses de la théorie financière (Bromwich, 2007 ; Ronen, 2008). De fait, la fair value ne peut prétendre à être fair et objective que si les marchés sont assez liquides et transparents pour que le prix de marché apparaisse comme reflétant à tout moment la réelle valeur des actifs qui s'y échangent. Adopter la fair value comme principe comptable universel revient donc à accepter les prémisses de la théorie financière selon lesquels les marchés d'actifs financiers sont des marchés efficients sur lesquels l'intensité des échanges garantit l'intégration très rapide de l'information dans les prix. L'objectif des régulateurs comptables promoteurs de la fair value était bien de rapprocher la comptabilité des marchés financiers, pour rompre avec la déconnexion entre prix de marché et coût historique, qui prévalait dans le système traditionnel. La rupture n'était pas mince, ce qui explique la chaleur des débats autour de cette notion. Adopter la fair value, c'était en effet donner un nouveau rôle à la comptabilité, un rôle en miroir des marchés financiers, qui, grâce à l'hypothèse d'efficience des marchés, pouvaient légitimement devenir la source première d'information, c'est-à-dire la base, pour l'ensemble des pratiques comptables. En ce sens, l'adoption de la notion de fair value en comptabilité internationale apparaît comme une illustration particulièrement forte de l'influence exercée par la théorie financière sur les pratiques et les cadres cognitifs régissant les marchés et les organisations (Davis, 2009 ; Whitley, 1986). Que cette méthode ait été appelée " fair » value témoigne de la dimension linguistique prise par cette influence. 
Une fois cette dimension linguistique reconnue, qu'en est-il du caractère performatif de l'adoption d'un vocable sous-tendu par un paradigme théorique ? L'adoption à l'échelle internationale de la fair value a changé en profondeur les pratiques comptables. Elle s'est heurtée à un certain nombre de difficultés pratiques, notamment lorsque l'hypothèse de marchés efficients n'étant guère réalisée, il a fallu recourir à différents expédients (dont les fameux modèles internes des banques par exemple) pour estimer ce que serait un prix de marché s'il en existait un. En outre, la fair value appliquée au bilan des banques est devenue, lors de la crise financière de 2007-2008, un facteur de pro-cyclicité auquel il a fallu apporter des correctifs, qui ont, à leur tour, fait l'objet de débats. Ainsi, on est loin de pouvoir considérer que l'adoption de la " fair " value ait eu des effets auto-réalisateurs univoques. En revanche, il est certain qu'elle a été un vecteur puissant de transformation des pratiques comptables et, partant, de l'influence de la théorie financière sur les pratiques et les réalités organisationnelles et marchandes. On peut voir dans l'adoption de la fair value un effet, particulièrement explicite, de la performativité des théories de la finance.

À travers l'analyse de l'impact de la recherche académique et de la théorie qu'elle produit sur les arrangements institutionnels, les normes sociales et le vocabulaire qui organisent la sphère financière, on peut donc prendre la pleine mesure de la performativité de la théorie financière, notamment en matière de régulation financière. Plus ou moins volontairement, sans le conceptualiser, en dehors de toute prévision consciente et de tout agenda précis qui lui serait propre, la théorie financière, dans le temps long, influe sur la régulation des marchés et des organisations qui relève de sa sphère d'analyse. II est notable qu'elle ne fasse pas retour sur ce phénomène. Observée et étudiée par d'autres - sociologues (MacKenzie, 2005 ; MacKenzie et Millo, 2003 ; Lépinay, 2011 ; Montagne, 2012), ethnologues et anthropologues (Ho, 2009 ; Miyazaki, 2007), spécialistes de la comptabilité (Power, 2010 ; Vollmer et al., 2009 ; Svetlova, 2012) ou de l'économie publique (Lockwood, 2015) -, la performativité de la théorie financière n'est pas prise en compte par la recherche académique en finance. Dans la dernière partie de cet article, nous proposons de réfléchir aux conséquences de cette situation, non pas concernant l'ensemble des relations entre la théorie financière et ses objets d'études, ce qui dépasserait le cadre de cet article, mais dans le domaine spécifique de la régulation financière.

\section{À quoi pourrait servir une théorie financière plus réflexive?}

La comptabilité est une discipline académique qui n'est pas sans proximité, au moins concernant son objet d'études, avec la finance. Or, ce qui frappe, lorsque l'on constate la manière dont la comptabilité prend en compte la performativité de ses savoirs et le silence absolu de la recherche en finance sur le même thème, c'est l'écart entre les deux disciplines en matière de réflexivité. Infiniment plus que la finance, la comptabilité, telle qu'elle se pratique académiquement, reprend à son compte la démarche méthodologique issue de la sociologie et de l'anthropologie (Bourdieu, 2001) qui consiste à appliquer les outils habituels de l'analyse non seulement à l'objet d'étude, mais aussi à son propre travail en tant que chercheur, ou à sa propre réflexion. La réflexivité suppose de renoncer à l'illusion de transparence dans la relation à l'objet d'analyse, d'expliciter les cadres sociaux des chercheurs, et 
de porter une attention particulière à l'influence que la présence ou la méthode du chercheur (un ethnologue par exemple) peut avoir sur les résultats qu'il obtient. Outil indispensable de l'arsenal de l'ethnologue ou de l'historien, qui doivent saisir d'où ils parlent pour mieux comprendre leur objet d'étude, la réflexivité n'est pas le fort de la recherche académique en finance. La raison en est sans doute que cette dernière s'identifie plus naturellement - mais non sans illusions (Rainelli-Le Montagner, 2003) - aux sciences de la nature qu'aux sciences sociales. Or, il n'est possible de se pencher sur la performativité de la théorie que si l'on reconnaît la validité du mouvement réflexif et si l'on renonce à concevoir son objet d'étude séparément de l'analyse qui en est faite. Pour " voir » qu'une théorie est performative, il faut être capable de ce mouvement, et reconnaître le profit qu'il peut y avoir à intégrer à l'analyse les présupposés, les perspectives, les impensés du chercheur d'une part et aussi les impacts que ce dernier peut avoir sur les pratiques. Ainsi, l'absence de reconnaissance, dans le champ de la finance académique, du caractère performatif des théories développées relève sans doute des réticences de ses pères fondateurs et de leurs successeurs à inscrire pleinement ce domaine de connaissances dans le champ des sciences sociales. Ce faisant, la recherche en finance se prive de l'outil « réflexivité » largement utilisé par d'autres, y compris sur son propre objet d'études.

La question qui se pose alors est de savoir si ce parti-pris méthodologique entraîne des conséquences négatives, qui justifieraient que l'on corrige le tir. Dans le domaine que nous analysons ici, celui de la régulation financière, l'inconvénient majeur de la non-réflexivité est de réduire la capacité de la recherche académique en finance à s'inscrire dans les débats de société que soulève cette question, souvent à l'occasion de crises financières qui peuvent, comme on le sait, avoir des conséquences économiques et sociales sévères. Ne pas intégrer les forces performatives de la théorie dans l'analyse limite cette dernière, comme l'illustre la citation d'Alan Greenspan au début de cet article. Reconnaître ne pas comprendre comment les choses ont pu arriver est, clairement, un aveu de faiblesse. Face aux débats soulevés par les crises et les questions qu'elles posent en matière de régulation financière, la non-réflexivité de la recherche académique en finance condamne celle-ci à une forme de mutisme, qui peut être dommageable pour son image dans la société. Tout se passe comme si la crise agissait en révélateur. Quelque chose, ou parfois le système tout entier, dont les principes de développements étaient soutenus, selon le mécanisme performatif décrit plus haut, par le paradigme de la finance théorique, dysfonctionne. Les dysfonctionnements constatés interrogent l'opinion publique mais l'absence de réflexivité de la discipline entrave largement la capacité de cette dernière à épouser ces interrogations. Des chercheurs étudient certes les questions de régulation (Benoit et al., 2017 ; Hendershott, Jones et Menkveld, 2011 ; Easley, Lopez de Prado et O'Hara, 2011 ; Chaboud, Chiquoine, Hjalmarsson et Vega, 2014 ; Hasbrouck et Saar, 2013 ; Duchin et Sosyura, 2014). Mais ils continuent de le faire dans le cadre du paradigme initial. On ne voit guère la finance comportementale, que certains identifient comme modifiant en profondeur le paradigme initial (Charreaux et Albouy, 2017), fournir pour l'instant d'assises vraiment nouvelles à ce type de débat. Bien plutôt, comme le notent Marti et Scherer (2016), les auteurs de la finance, tenus par le cadre paradigmatique de la finance académique, ne gardent finalement, comme aune unique de la qualité de la régulation, que la question de l'efficience des marchés. Or, l'efficience ayant partie liée avec l'activité sur les marchés, cela les conduit immanquablement à préférer plus d'échange à moins d'échange et à accueillir 
favorablement toute innovation technologique de nature à les intensifier. II s'agit finalement de proposer plus de la même chose, sans disposer de la capacité - que seule permettrait une réflexivité construite sur une prise en compte des effets performatifs du paradigme de référence - de prendre en compte de potentiels effets négatifs, par exemple sur la stabilité des marchés ou sur les incitations à la duperie ou à la fraude générées par le développement de marchés nouveaux (Duffie et Stein, 2015).

On peut tenir, à l'instar des pères fondateurs du paradigme de la finance moderne, que la stricte observation des frontières du paradigme est une bonne chose. On peut aussi, à l'aune de la dernière crise financière globale, des questions qu'elle a soulevées en matière de régulation et de l'impuissance des régulateurs à imprimer des tournants forts dans la régulation actuelle (Munzer, 2016 ; Benoit et al., 2017 ; Philippon, 2016 ; Duffie et Stein, 2015), se demander si la posture non réflexive ne produit pas à terme une usure, qui rend la recherche académique de moins en moins apte à élaborer des réponses convaincantes sur les questions de régulation des marchés. On peut se demander si ce repli sur le paradigme d'origine ne la condamne pas à ne promouvoir que des changements techniques ou technocratiques, et incrémentaux. II ne s'agirait pas alors du complet mutisme décrit plus haut, mais de sérieuses limitations à l'innovation conceptuelle tant la capacité de la réalité financière à sortir du cadre de la théorie, notamment sous l'effet de facteurs performatifs cumulatifs ${ }^{6}$, créerait, à force de n'être pas reconnue, un " monstre » à peu près impossible à maîtriser (Acharya et al., 2010 ; Schneiberg et Bartley, 2010 ; Philippon, 2016). Par sa taille, sa complexité et les coûts de coordination immenses induits par sa régulation au niveau mondial, le système financier donne déjà l'impression d'être devenu impossible à réformer. Et certain auteurs (Philippon, 2016), devant l'ampleur de la tâche et dans une sorte d'aveu d'impuissance, semblent s'en remettre aux seules évolutions technologiques de l'industrie financière (le développement des fintechs par exemple) pour la création d'un meilleur système financier que celui dont on dispose aujourd'hui.

En quoi une meilleure prise en compte de la performativité de la théorie et, partant, une plus grande réflexivité aideraient-elles les chercheurs en finance à dépasser cette forme d'impuissance ? Tout d'abord, on peut imaginer qu'étudier les effets induits de la théorie est susceptible de produire des pistes de recherche nouvelles. Ainsi la question de la stabilité des marchés, que les académiques en finance ne traitaient guère, a-t-elle été investie par nombre de sociologues plus disposés à exercer leur réflexivité sur les outils de la finance. En s'interrogeant sur la capacité des algorithmes à réaliser des tâches jusque-là traitées par des acteurs humains, certains de ces sociologues ont montré que les algorithmes avaient tendance à amoindrir les capacités des acteurs de marchés à donner du sens aux événements lorsque les marchés sont turbulents (MacKenzie et al., 2012) et que cette perte de sens avait tendance à accroître la volatilité des marchés (Beunza et Millo, 2015). Cela les conduit à se prononcer, contrairement aux auteurs s'inscrivant dans le champ de la finance, qui voient dans le trading haute fréquence une source d'efficience accrue, pour une régulation du recours à cette technique, à cause de ses

\footnotetext{
${ }^{6}$ Thiemann et Lepoutre (2017) utilisent le terme de creative compliance pour montrer comment la règle est toujours contournée, détournée par les acteurs s'appuyant sur les ressorts de la théorie financière.
} 
effets sur la stabilité des marchés. En se penchant sur la manière dont les innovations financières ont pu être utilisées dans différents pays pour échapper à la régulation, Thiemann et Lepoutre (2017) mettent en évidence l'importance des réseaux qui gouvernent la régulation des marchés, la manière dont les institutions de marchés sont prises dans ces réseaux et la pertinence de l'analyse des relations de pouvoir qui gouvernent les structures de régulation. Ils soulignent, notamment, l'importance de la manière dont les règles sont interprétées et insistent plus spécifiquement sur la question de l'architecture de cette interprétation. II existe, ainsi qu'ils le montrent, un enjeu de pouvoir entre régulateurs et régulés des marchés financiers, les régulateurs affaiblissant leurs positions lorsqu'ils dépendent, comme c'est souvent le cas, des propositions des régulés concernant l'interprétation et la mise en œuvre des règles décidées au cours de processus complexes auxquels tous concourent à des degrés divers. Ces exemples illustrent la manière dont l'étude des effets performatifs de la théorie sur les pratiques financières peut générer des connaissances nouvelles et ouvrir des pistes de recherche inaperçues jusque-là, tout en enrichissant les dimensions de ce que serait le bien commun en matière de régulation financière. S'ouvrir à la réflexivité permettrait sans doute à la recherche en finance de cesser de borner son horizon à la poursuite de l'unique objectif d'améliorer l'efficience des marchés, comprise dans un sens assez limité. Elle pourrait alors s'emparer d'autres questions comme celle de la stabilité des marchés - quel est le niveau de stabilité souhaitable ? comment y parvient-on ? -, ou celle des incitations à la fraude ou au dépassement des règles qu'un certain type de système financier engendre (Duffie et Stein, 2015 ; Thiemann et Lepoutre, 2017).

En second lieu, une approche plus réflexive des effets performatifs de la théorie ou de pratiques financières qui s'y rattachent, en donnant droit de cité à des questions dépassant la seule problématique de l'efficience, conduit à considérer le point de vue de nouvelles parties prenantes intéressées au débat. En étudiant le chemin qui existe, dans la réalité, entre l'innovation financière produite par la théorie et la création d'un véritable marché pour cette innovation, des chercheurs (MacKenzie et Millo, 2003 ; Beunza et Stark, 2012 ; Lépinay, 2011 ; Huault et Rainelli-Le Montagner, 2009 ; Thiemann et Lepoutre, 2017) ont montré les enjeux de pouvoir, les intérêts de parties prenantes variées et interrogé la question des institutions de la finance. Ces travaux, qui admettent le caractère performatif de la théorie financière et qui se fondent sur un parti-pris réaliste (que se passe-t-il réellement, en pratique, sur les marchés financiers?), incluent naturellement les parties prenantes rencontrées en chemin. Ils sont ainsi de nature à nourrir une réflexion enrichie sur ce que serait une régulation financière souhaitable, du point de vue du bien commun. On peut envisager qu'en adoptant cette posture de reconnaissance de la performativité de la théorie et de parti-pris réaliste, la théorie financière sortirait du rôle où elle se cantonne lorsqu'elle reste crispée sur son paradigme originel. Elle pourrait ainsi se rendre plus apte à soutenir une réflexion non seulement plus diverse et incluant plus de parties prenantes, mais aussi plus pertinente, sur la régulation d'une sphère financière en perpétuelle évolution et très différente de celle qui prévalait lorsque les bases du paradigme actuel ont été posées.

\section{Conclusion : juger a priori de l'impact de la recherche}

Notre recension de l'impact qu'a pu avoir la recherche en finance ces dernières décennies, notamment sur la régulation financière, nous conduit finalement à 
l'interrogation suivante : qu'y a-t-il à retenir des mécanismes identifiés qui puisse nous être utile pour la suite?

Ce que nous apprend sans doute la cassure que constitue la crise financière des années 2007-2008, c'est qu'il faut être prudent en la matière et reconnaître I'historicité des objectifs poursuivis par un paradigme donné. Contrairement à ce que I'on a pu croire au temps de la finance triomphante des années 1990-2000, il ne nous semble plus si certain qu'il suffise aux producteurs de savoir de favoriser une recherche à fort impact sur les pratiques, une recherche débouchant sur des innovations financières et participant à la croissance de tout un secteur pour être certain de bien faire. II semble dès lors que, pour qui souhaiterait adopter une perspective normative sur ce que serait une bonne théorie, une théorie bonne à bâtir, il faille, à la manière de Marti et Scherer (2016), accepter la pluralité des objectifs potentiels. Se concentrant sur l'innovation financière et à partir de l'agir communicationnel proposé par Habermas, ces deux auteurs mettent par exemple en avant les objectifs d'efficience, de stabilité et de justice sociale et proposent d'interroger l'impact des innovations et de la régulation financières sur ces trois dimensions. Ils incitent les chercheurs à sortir des limites des théories existantes, qui, par nature, tendent à exclure cette pluralité de dimensions. Dès lors que l'on veut normativement définir ce que serait une "bonne » recherche en finance, il faut sans doute admettre qu'au-delà des acteurs économiques concernés, l'organisation du système économique ou financier concerne la société tout entière et interroge la notion de bien commun. En finance comme en matière de normalisation comptable, sujets techniques s'il en est, les divergences d'intérêts sont en réalité des questions politiques, ce que leur cantonnement dans des sphères technocratiques, excluant presque entièrement le débat démocratique, fait perdre de vue (Ramanna, 2015). D'où il découle qu'une recherche désirable est peut-être d'abord et avant tout une recherche plurielle, apte à adopter des perspectives variées, et parfois contraires, et à sortir du carcan performatif des paradigmes qui ont pu faire son succès.

Reconnaître la performativité de la théorie financière et adopter par suite une position réflexive sur la discipline nous semble être un moyen potentiellement efficace de renouveler les perspectives, de s'assigner des objectifs variés et de se donner une chance d'intégrer les intérêts de parties prenantes négligées dans certains paradigmes. Ce que nous apprend l'Histoire, c'est que sans cette pluralité d'objectifs et de perspectives, le risque est grand de développer une recherche dont l'impact réel peut se révéler a posteriori décevant, au mieux pour certaines parties prenantes seulement, au pire pour l'ensemble de la société et le bien commun général.

\section{Bibliographie}

ABRAHAMSON E., BERKOWITZ H., \& DUMEZ H. (2016), "A more relevant approach to relevance in Management Studies: an essay on performativity", Academy of Management Review, 41(2), 367-381.

ACHARYA V. V., COOLEY T. F., RICHARDSON M. P. \& WALTER I. (2010), Regulating Wall Street: The Dodd-Frank Act and the new architecture of global finance (Vol. 608), John Wiley \& Sons. 
AGLIETTA M. \& REBÉRIOUX A. (2004), Dérives du capitalisme financier, Paris, Albin Michel.

ARNOLD T., BUTLER A. W., FALCON CRACK T. \& ALTINTIG A, "Impact: What Influences Finance Research?", The Journal of Business 76 (2) (2003): pp. 343-361.

Conseil d'analyse économique, ARTUS, P., BETBĖZE J.-P., de BOISSIEU C. \& CAPELLE-BLANCARD G. (2008), La Crise des subprimes, La Documentation française.

BALDVINSDOTTIR G., MITCHELL F. \& NØRREKLIT H. (2010), "Issues in the relationship between theory and practice in management accounting", Management Accounting Research, 21(2), pp. 79-82.

BARNES B. (1983), "Social life as bootstrapped induction", Sociology, 17, pp. 524545.

BENOIT S., COLLIARD J. E., HURLIN C. \& PÉRIGNON C. (2017), "Where the risks lie: A survey on systemic risk", Review of Finance, 21(1), pp. 109-152.

BERNSTEIN P. L. (1993), Capital ideas: the improbable origins of modern Wall Street, Simon and Schuster.

BEUNZA D. \& MILLO Y. (2015), "Blended automation: Integrating algorithms on the floor of the New York Stock Exchange”, SRC Discussion Paper n³8, May.

BEUNZA D. \& STARK D. (2012), "From dissonance to resonance: cognitive interdependence in quantitative finance", Economy and Society, 41(3), pp. 383-417.

BLACK F. \& SCHOLES M. (1973), "The pricing of options and corporate liabilities", The Journal of political economy, pp. 637-654.

BOURDIEU P. (2001), Science de la science et réflexivité, Paris, Raisons d'agir.

BROIHANNE M. H., MERLI M. \& ROGER P. (2004), Finance comportementale, Economica.

BROMWICH M. (2007), Fair values: imaginary prices and mystical markets, pp. 4668, London, Routledge.

BRYAN D. \& RAFFERTY M. (2006), Capitalism with derivatives, New York, Plagrave Macmillan.

BRYAN D. \& RAFFERTY M. (2014), "Financial Derivatives as Social Policy beyond Crisis", Sociology, 48(5), pp. 887-903.

BUTLER J. (1997), Excitable Speech. A Politics of the Performative, New York, Routledge. 
CABANTOUS L. \& GOND J.-P. (2011), "Rational decision making as performative praxis: explaining rationality's éternel retour", Organization Science, 22(3), pp. 573586.

CALLON M. (1998), The Laws of the Markets, Oxford, Blackwell.

CALLON M. (2007), "What does it mean to say that economics is performative?", Do economists make markets? On the performativity of economics, MACKENZIE D., MUNIESA F. \& SIU L. (eds), Princeton University Press, Princeton.

CHABOUD A. P., CHIQUOINE B., HJALMARSSON E. \& VEGA C. (2014), "Rise of the machines: Algorithmic trading in the foreign exchange market", The Journal of Finance, 69(5), pp. 2045-2084.

CHARREAUX G. \& ALBOUY M. (2017), « La construction de la théorie financière moderne : de la finance néoclassique à la finance néo-institutionnelle et comportementale. Une introduction aux grands auteurs en finance », in ALBOUY M. \& CHARREAUX G. (dir.), Les grands auteurs en finance, $2^{\mathrm{e}}$ édition, pp. 5-55. (https://hal-univ-bourgogne.archives-ouvertes.fr/hal-01621313)

CHIAPELLO E. \& WALTER C. (2016), "The Three Ages of Financial Quantification: A conventionalist Approach to the Financiers Metrology", Historical Social Research 41(2), pp. 155-177.

DAVIS G. F. (2009), Managed by the markets: How finance reshaped America, Oxford University Press.

DAVIS G. F. (2015), "Editorial essay: what is organizational research for?", Administrative Science Quarterly, 60(2), pp. 179-188.

DAVIS G. F., DIEKMANN K. A. \& TINSLEY C. H. (1994), "The decline and fall of the conglomerate firm in the 1980s: The deinstitutionalization of an organizational form". American sociological review, pp. 547-570.

DE SCHEEMAEKERE X. (2009), “The epistemology of modern finance”, The Journal of Philosophical Economics, 2(2), pp. 99.

DERRIDA J. (1979), Signature event context ? Glyph, 1, pp. 172-197.

DUCHIN R. \& SOSYURA D. (2014), "Safer ratios, riskier portfolios: Banks' response to government aid”, Journal of Financial Economics, 113(1), pp. 1-28.

DUFFIE D. \& STEIN J. C. (2015), "Reforming LIBOR and other financial market benchmarks”, Journal of Economic Perspectives, 29(2), pp. 191-212.

EASLEY D., DE PRADO M. M. L. \& O'HARA M. (2011), "The microstructure of the 'flash crash': Flow toxicity, liquidity crashes, and the probability of informed trading", Journal of Portfolio Management, 37(2), p. 118. 
FERRARO F., PFEFFER J. \& SUTTON R. I. (2005), "Economics language and assumptions: How theories can become self-fulfilling", Academy of Management Review, 30(1), pp. 8-24.

FLIGSTEIN N. (1993), The Transformation of corporate control, Harvard University Press.

GOND J.-P., CABANTOUS L., HARDING N. \& LEARMONTH M. (2015), "What do we mean by performativity in organizational and management theory? The uses and abuses of performativity", International Journal of Management Reviews.

HARDING N. (2003), The Social Construction of Management, London, Routledge.

HARGIE O., STAPLETON K. \& TOURISH D. (2010), "Interpretations of CEO public apologies for the banking crisis: attributions of blame and avoidance of responsibility", Organization, 17(6), pp. 721-742.

HASBROUCK J. \& SAAR G. (2013), "Low-latency trading”, Journal of Financial Markets, 16(4), pp. 646-679.

HENDERSHOTT T., JONES C. M. \& MENKVELD A. J. (2011), "Does algorithmic trading improve liquidity?", The Journal of Finance, 66(1), pp. 1-33.

HO K. (2009), Liquidated: an ethnography of Wall Street, Duke University Press.

HUAULT I. \& RAINELLI-LE MONTAGNER H. (2009), "Market shaping as an answer to ambiguities: the case of credit derivatives", Organization Studies, 30(5), pp. 549$575 ; 887-903$.

HUAULT I. \& RAINELLI-WEISS H. (2013), "The connexionist nature of modern financial markets: from a domination to a justice order", New Spirits of Capitalism? Crises, Justifications, and Dynamics, pp. 181-205.

JENSEN M. C. (1978), "Some anomalous evidence regarding market efficiency", Journal of financial economics, 6(2/3), pp. 95-101.

JENSEN M. C. \& MECKLING W. H. (1976), "Theory of the firm: Managerial behavior, agency costs and ownership structure", Journal of financial economics, 3(4), pp. 305360 .

KAPLAN R. S. (2011), "Accounting scholarship that advances professional knowledge and practice", The Accounting Review, 86(2), pp. 367-383.

KINDLEBERGER C. P. (2001), Manias, panics and crashes, Palgrave Macmillan.

KUHN T. S. (1972), La Structure des révolutions scientifiques, Flammarion.

LEARMONTH M. (2005), "Doing things with words: the case of management and administration", Public Administration, 83, pp. 617-637. 
LÉPINAY V. A. (2011), Codes of finance: Engineering derivatives in a global bank. Princeton University Press.

LIPUMA E. \& LEE B. (2004), Financial derivatives and the globalization of risk, Duke University Press.

LOCKWOOD E. (2015), "Predicting the unpredictable: Value-at-risk, performativity, and the politics of financial uncertainty", Review of International Political Economy, 22(4), pp. 719-756.

LYDENBERG S. (2014), "Reason, rationality, and fiduciary duty", Journal of business ethics, 119(3), pp. 365-380.

MACKENZIE D. (2006), An Engine, Not a Camera: How Financial Models shape Markets, Cambridge, MIT Press.

MACKENZIE D. \& MILLO Y. (2003), "Negotiating a Market, Performing Theory: The Historical Sociology of a Financial Derivatives Exchange", American Journal of Sociology.

MACKENZIE D. \& SPEARS T. (2014), “'The formula that killed Wall Street': The Gaussian copula and modelling practices in investment banking", Social Studies of Science, 44(3), pp. 393-417.

MACKENZIE D., BEUNZA D., MILLO Y., \& PARDO-GUERRA J.-P. (2012), "Drilling through the Allegheny Mountains: Liquidity, materiality and high-frequency trading", Journal of cultural economy, 5(3), pp. 279-296.

MARKOWITZ H. (1952), "Portfolio selection", The Journal of Finance, 7(1), pp. 7791.

MARTI E. \& SCHERER A. G. (2016), "Financial regulation and social welfare: The critical contribution of management theory", Academy of Management Review, 41(2), pp. 298-323.

MARTIN D. (2015), « Domestication sociologique d'un produit financier » in CHAMBOST I. et al. (2015), La Fabrique de la finance, Pour une approche interdisciplinaire, Presses Universitaires du Septentrion, coll. Capitalismes - éthique institutions, pp. 35-42.

MERTON R.C. (1995), "Influence of mathematical models in finance on practice: Past, present and future", Financial Practice and Education, 5, pp. 7-15.

MILLER P. \& POWER M. (2013), "Accounting, organizing, and economizing: Connecting accounting research and organization theory", Academy of Management Annals, 7(1), pp. 557-605.

MIYAZAKI H. (2007), "Between arbitrage and speculation: an economy of belief and doubt”, Economy and Society, 36(3), pp. 396-415. 
MOEHRLE S., ANDERSON K., AYRES F., BOLT-LEE C., DEBRECENY R., DUGAN M., HOGAN C., MAHER M. and PLUMMER E. (2009), "The impact of academic accounting research on professional practice: an analysis by the AAA Research Impact Task Force", Accounting Horizons, Vol. 23, n4, p. 411.

MONTAGNE S. (2012), «Investir avec prudence : les usages d'un impératif juridique par les acteurs du capitalisme financiarisé », Sociologie du travail, 54(1), pp. 92-111.

MORGAN G. (2008), « Market formation and governance in international financial markets: The case of OTC derivatives ${ }^{1}$ », Human Relations, 61(5), pp. 637-660.

MUNIESA F. (2014), The provoked economy: Economic reality and the performative turn, Routledge.

MUNIR K.A. (2011), "Financial crisis 2008-9: what does the silence of institutional theorists tell us?", Journal of Management Inquiry, 20(2), 114-117.

MUNZER M. (2016), Financial market regulation in the aftermath of the financial crisis: three essays on structural banking reforms (Doctoral dissertation, Strasbourg).

MYERS S. C. (2015), "Finance, Theoretical and Applied", Annual Review of Financial Economics, 7, pp. 1-34.

ORLÉAN A. (2009), De l'euphorie à la panique : penser la crise financière (Vol. 16), Éditions Rue d'Ulm.

PARKER L. D., Guthrie J. \& Linacre S. (2011), "The relationship between academic accounting research and professional practice", Accounting, Auditing \& Accountability Journal, 24(1), pp. 5-14.

PHILIPPON T. (2016), "The fintech opportunity”, (No. w22476), National Bureau of Economic Research.

PICKERING A. (1995), The Mangle of Practice: Time, Agency, and Science. Chicago, IL: University of Chicago Press.

POWER M. (2010), "Fair value accounting, financial economics and the transformation of reliability", Accounting and Business Research, 40(3), pp. 197-210.

PRYKE M. \& ALLEN J. (2000), "Monetized time-space: derivatives-money's' new imaginary'?", Economy and Society, 29(2), pp. 264-284.

RAINELLI-WEISS H. \& HUAULT I. (2016), "Business as usual in Financial Markets? The creation of incommensurables as institutional maintenance work", Organization Studies.

RAINELLI-Le MONTAGNER H. (2003), Nature et fonctions de la théorie financière: quelques réflexions, Presses universitaires de France. 
RAMANNA K. (2015), "Thin Political Markets", California Management Review, 57(2), pp. 5-19.

ROBERTS J. H., KAYANDE U. \& STREMERSCH S. (2014), "From academic research to marketing practice: Exploring the marketing science value chain"? International Journal of Research in Marketing, 31(2), pp. 127-140.

Ronen J. (2008), "To fair value or not to fair value: a broader perspective", Abacus, 44(2), pp. 181-208.

SCHNEIBER M. \& BARTLEY T. (2010), "Regulating or redesigning finance? Market architectures, normal accidents, and dilemmas of regulatory reform", Research in the Sociology of Organizations, 30A, pp. 281-307.

SEARLE J. (1969), Speech Acts: An Essay in the Philosophy of Language, Cambridge: Cambridge University Press.

SHARPE W. F. (1964), "Capital asset prices: A theory of market equilibrium under conditions of risk", The Journal of Finance, 19(3), pp. 425-442.

SHILLER R. J. (2012), Finance and the good society (Reprint), Princeton, Princeton University Press.

SVETLOVA E. (2012), "On the performative power of financial models", Economy and Society, 41(3), pp. 418-434.

THIEMANN M. \& LEPOUTRE J. (2017), "Stitched on the Edge: Rule Evasion, Embedded Regulators, and the Evolution of Markets", American Journal of Sociology, 122(6), pp. 1775-1821.

VOLLMER H., MENNICKEN A. \& PREDA A. (2009), "Tracking the numbers: Across accounting and finance, organizations and markets", Accounting, Organizations and Society, 34(5), pp. 619-637.

Whitley R. (1986), "The transformation of business finance into financial economics: The roles of academic expansion and changes in US capital markets", Accounting, Organizations and Society, 11(2), pp. 171-192.

Zingales L. (2015), “Presidential Address: Does Finance Benefit Society?”. The Journal of Finance, 70(4), pp. 1327-1363.

ZORN D. M. (2004), "Here a chief, there a chief: The rise of the CFO in the American firm", American Sociological Review, 69(3), pp. 345-364.

ZORN D., DOBBIN F., DIERKES J. \& KWOK M. S. (2014), "Managing investors: How financial markets reshaped the American firm", Paper ssrn. 